\title{
Numerical dosimetric calculations for in vitro field expositions in the THz frequency range
}

\author{
C. Jastrow, T. Kleine-Ostmann, and T. Schrader \\ Physikalisch-Technische Bundesanstalt, Braunschweig and Berlin, Germany
}

\begin{abstract}
Field exposition experiments have been initiated by the German Federal Office for Radiation Protection (Bundesamt für Strahlenschutz - BfS) to examine genotoxic effects of $\mathrm{THz}$ radiation in vitro. Two different human skin cell types are exposed to continuous-wave radiation at six distinct frequencies between $100 \mathrm{GHz}$ and $2.52 \mathrm{THz}$ originating from different sources of $\mathrm{THz}$ radiation under defined environmental conditions. The cell containers are irradiated with free space power flux densities between $0.1 \mathrm{~mW} / \mathrm{cm}^{2}$ and $2 \mathrm{~mW} / \mathrm{cm}^{2}$ measured traceable to the SI units. For meaningful results, dosimetric calculations using the finite differences time-domain method have been performed in order to access the fields and consequently the specific absorption rate (SAR) in the cell layer.
\end{abstract}

\section{Introduction}

With the increasing amount of applications utilizing THz radiation appearing on the market (e.g. communication links, Piesiewicz et al., 2007, spectroscopy and quality control inspection systems, and security screening systems, Siegel, 2002) the question of health protection in non-ionizing electromagnetic fields arises for sub-mm radiation, also (KleineOstmann et al., 2006). Like in the frequency range below, it is still not clear whether non-ionizing RF radiation below the thermal damage threshold could cause detrimental effects in living organisms. The International Commission for NonIonizing Radiation Protection (ICNIRP) limits the power flux density for general public exposure for the frequency range between $2 \mathrm{GHz}$ and $300 \mathrm{GHz}$ to $1 \mathrm{~mW} / \mathrm{cm}^{2}$ (ICNIRP, 1998 , Physical Agents Directive 2004/40/EC, 2004, NiSG, 2009). The limit is based on the thermal damage threshold which

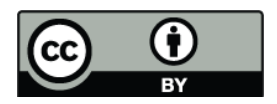

Correspondence to: T. Kleine-Ostmann (thomas.kleine-ostmann@ptb.de) (a)

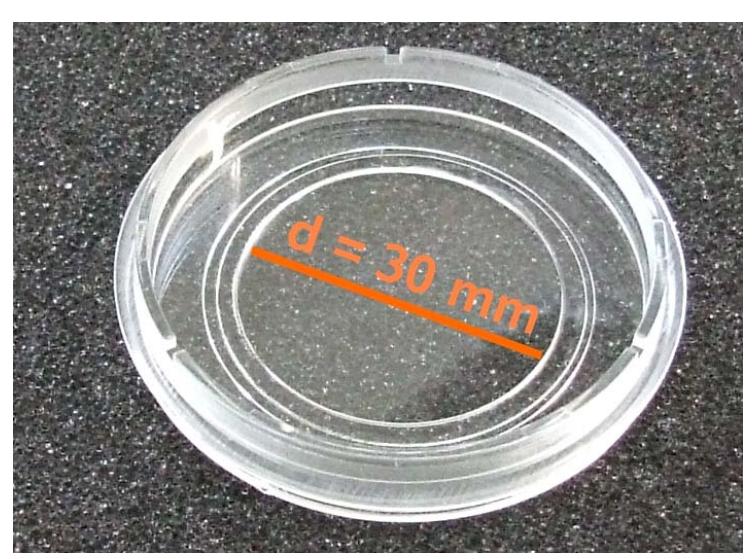

(b)

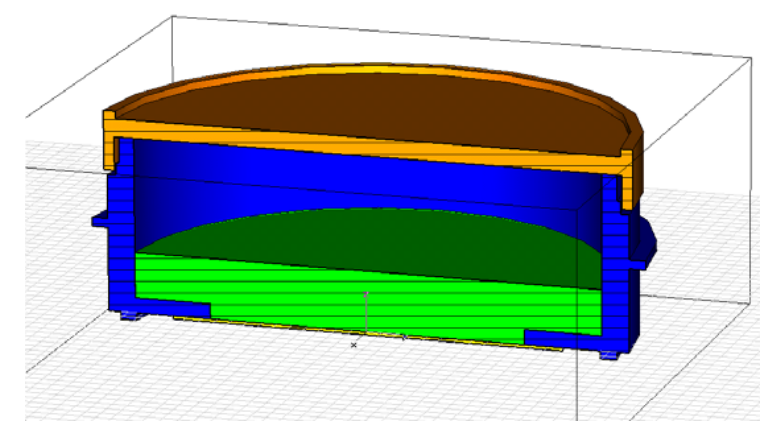

Fig. 1. (a) Sample container for field exposition experiments with skin cell lines (lid removed). (b) Computer model (CST Microwave Studio) for SAR calculation in a cell container filled with culture medium.

has been examined extensively in the microwave frequency range, only. Above $300 \mathrm{GHz}$ no such limits for public exposure exist and safety limits concern laser radiation, only. Depending on the specific laser source, the safety limits are in the range between $1 \mathrm{~mW} / \mathrm{cm}^{2}$ and $100 \mathrm{~mW} / \mathrm{cm}^{2}$ (ANSI,

Published by Copernicus Publications on behalf of the URSI Landesausschuss in der Bundesrepublik Deutschland e.V. 


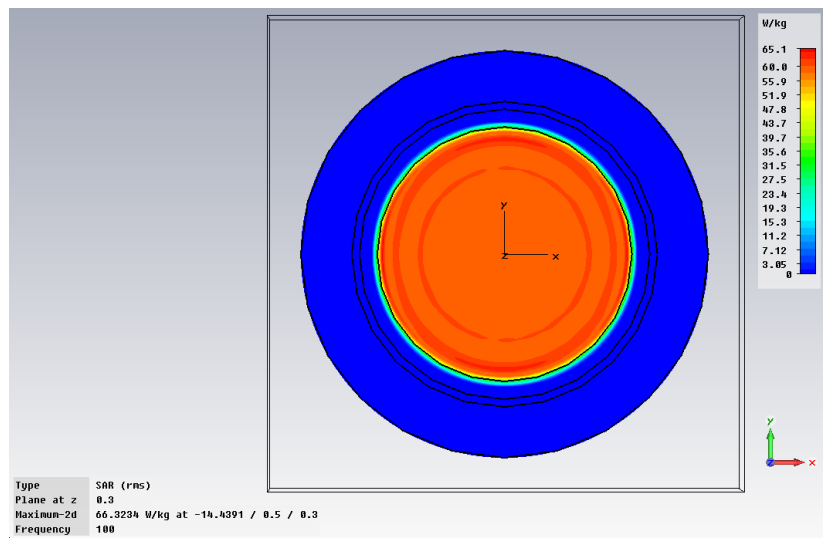

Fig. 2. Results of the SAR calculation (0.001 g RMS) at $100 \mathrm{GHz}$ in the cell layer for a plane wave with $10 \mathrm{~mW} / \mathrm{cm}^{2}$ travelling in the $\mathrm{z}$-direction. The cut plane is located $100 \mu \mathrm{m}$ above the bottom. Results are valid both for isothermic and open boundary conditions.

2007). An early study by Berry et al. (2002) shows that human exposure due to existing $\mathrm{THz}$ imaging systems is usually well below $1 \mathrm{~mW} / \mathrm{cm}^{2}$. However, the non-thermal effects of non-ionizing radiation are still discussed controversially. In the $\mathrm{THz}$ frequency range, only one comprehensive study exists so far (THz-Bridge, 2004). KorensteinIlan et al. (2008) found increased genomic instability in human lymphocytes for exposure to $100 \mathrm{GHz}$ radiation well below the safety limit $\left(0.031 \mathrm{~mW} / \mathrm{cm}^{2}\right.$ in culture medium). Initiated by the BfS field exposition experiments to examine genotoxic effects of $\mathrm{THz}$ radiation in vitro are underway (Kleine-Ostmann et al., 2009). Under defined environmental conditions, two different human skin cell types are exposed to continuous-wave radiation at distinct frequencies between $100 \mathrm{GHz}$ and $2.52 \mathrm{THz}$ originating from different sources of $\mathrm{THz}$ radiation. The cell containers are irradiated with free space power densities between $0.1 \mathrm{~mW} / \mathrm{cm}^{2}$ and $2 \mathrm{~mW} / \mathrm{cm}^{2}$ measured traceable to the SI units (Kleine-Ostmann et al., 2008). In order to quantify and reliably evaluate the stress imposed on the living cells, it is necessary to determine the SAR within a monolayer of cells covered by culture medium in a cell container. In this contribution we show first dosimetric calculations to quantify the power introduction into the cell layer. The numerical calculations have been performed using the finite-differences time-domain method as implemented in the program CST Microwave Studio (2009). A detailed geometric model of the sample container and realistic dielectric properties of the materials are used to obtain reliable SAR values at $100 \mathrm{GHz}$. The results indicate that heating effects probably cannot be neglected at higher exposure levels when evaluating the outcome of the experiments. The calculated heat introduction is in good compliance with the temperature profile measured during field exposition using a fibre-coupled thermometer.

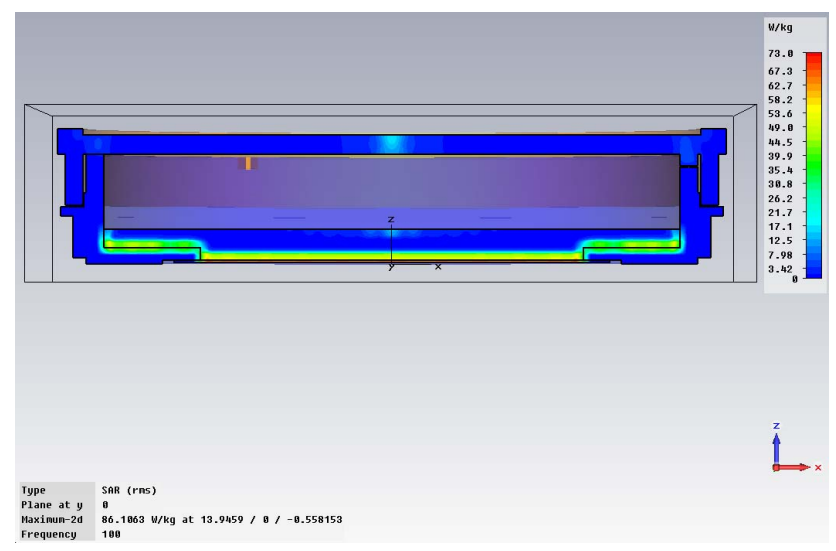

Fig. 3. Results of the SAR calculation $(0.001 \mathrm{~g} \mathrm{RMS})$ at $100 \mathrm{GHz}$ in the cell container for a plane wave with $10 \mathrm{~mW} / \mathrm{cm}^{2}$ travelling in the $\mathrm{z}$-direction. The cut plane is a cross section through the center. Results are valid both for isothermic and open boundary conditions.

\section{Field exposition experiments}

In the project, two different human skin cell types, i.e. the Human adult low Calcium Temperature (HaCaT) keratinocyte cell line and primary dermal fibroblasts, are exposed to $\mathrm{THz}$ radiation, since the penetration depth into the human body above $100 \mathrm{GHz}$ is well below $1 \mathrm{~mm}$ (THzBridge, 2004). The cells are cultivated in culture medium (Dulbecco's Modified Eagle Medium - DMEM) in a custom built sample container (ibidi GmbH) as shown in Fig. 1. The bottom of this container consists of a $200 \mu$ m thick foil which is transparent in the frequency range of interest (due to its low dielectric loss angle) on which the cells are fixed as a monolayer in a spot of $12 \mathrm{~mm}$ diameter in the center. The cells are irradiated from below in a modified incubator at six distinct frequencies of $100 \mathrm{GHz}, 130 \mathrm{GHz}, 385 \mathrm{GHz}, 604 \mathrm{GHz}$, $1.63 \mathrm{THz}$ and $2.52 \mathrm{THz}$ to verify the results from the previous study and to cover a larger part of the $\mathrm{THz}$ frequency range. Four different sources of $\mathrm{THz}$ radiation are used to irradiate the exposition zone with a $2 \mathrm{~cm}$ diameter Gaussian beam (full width half maximum) from below, respectively: a frequency multiplier cascade at $100 \mathrm{GHz}$, a Gunn oscillator at $130 \mathrm{GHz}$, a backward-wave oscillator at $385 \mathrm{GHz}$ and a farinfrared gas laser at $604 \mathrm{GHz}, 1.63 \mathrm{THz}$ and $2.52 \mathrm{THz}$. For each source, three power flux densities below $\left(0.1 \mathrm{~mW} / \mathrm{cm}^{2}\right)$, at $\left(1 \mathrm{~mW} / \mathrm{cm}^{2}\right)$ and above $\left(2 \mathrm{~mW} / \mathrm{cm}^{2}\right)$ the limit are chosen for the exposure. All power densities will be measured traceable to the SI units in order to allow for a precise and reliable assessment of the exposure dose. The traceable measurements were initially performed using a bolometer calibrated in front of a black body radiation source (Kleine-Ostmann et al., 2008). Later, to improve the reliability, a gas pressure membrane sensor traceable to an AC voltage (Thomas Keating Instruments, 2009) and a pyro-electric detector calibrated with a cryogenic substitution radiometer (Werner et al., 2009) were used. 


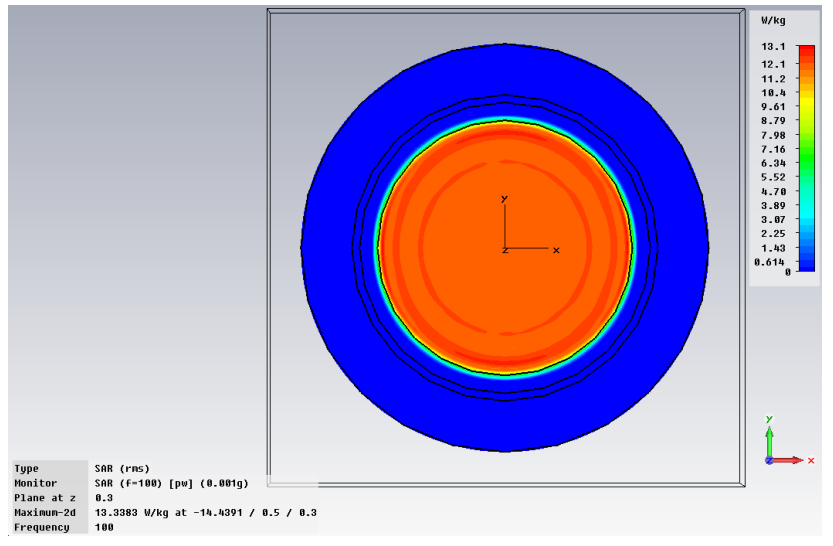

Fig. 4. Results of the SAR calculation (0.001 g RMS) at $100 \mathrm{GHz}$ in the cell layer for a plane wave with $2 \mathrm{~mW} / \mathrm{cm}^{2}$ travelling in the z-direction. The cut plane is located $100 \mu \mathrm{m}$ above the bottom. Results are valid both for isothermic and open boundary conditions.

During exposition, the sample containers are kept at defined environmental conditions of approximately $37^{\circ} \mathrm{C}$ and $5 \% \mathrm{CO}_{2}$ content of the atmosphere in a modified incubator as required by the cells. The temperature of the incubator is reduced to $36^{\circ} \mathrm{C}(1 \mathrm{~K}$ below the targeted value) to account for a certain heating of the cells during illumination with $\mathrm{RF}$ radiation. All experimental parameters are permanently monitored during the exposition experiments. Two different exposure times of $2 \mathrm{~h}$ and $8 \mathrm{~h}$ are chosen. The comet assay is used as biological end point for strand breaks (Singh, 1988), whereas the micronucleus test is chosen to search for possible aneugenic and clastogenic effects (Matter and Schmid, 1971). The validity of the results is guaranteed by positive controls, sham exposition experiments and a blinded experimental procedure. In order to obtain statistically significant results, four independent experimental series are conducted. Three different independent exposition series will be performed to validate the results from an initial run. From each exposed cell container, two different samples will be taken for the evaluation of the end points.

\section{Dosimetric calculations}

First dosimetric calculations to quantify the power introduction into the cell layer have been performed using the finite-differences time-domain method as implemented in the program CST Microwave Studio (2009). Due to the small vacuum wavelength of the $\mathrm{THz}$ radiation compared to the size of the cell container ranging from $3 \mathrm{~mm}$ at $100 \mathrm{GHz}$ to $0.119 \mathrm{~mm}$ at $2.52 \mathrm{THz}$, the calculations are strongly limited by computational resources. With a double quad-core $3.2 \mathrm{GHz}$ processor machine and $64 \mathrm{GByte}$ RAM the calculations were restricted to approximately 140 million voxels. SAR calculations with our model using 35 million voxels (re-

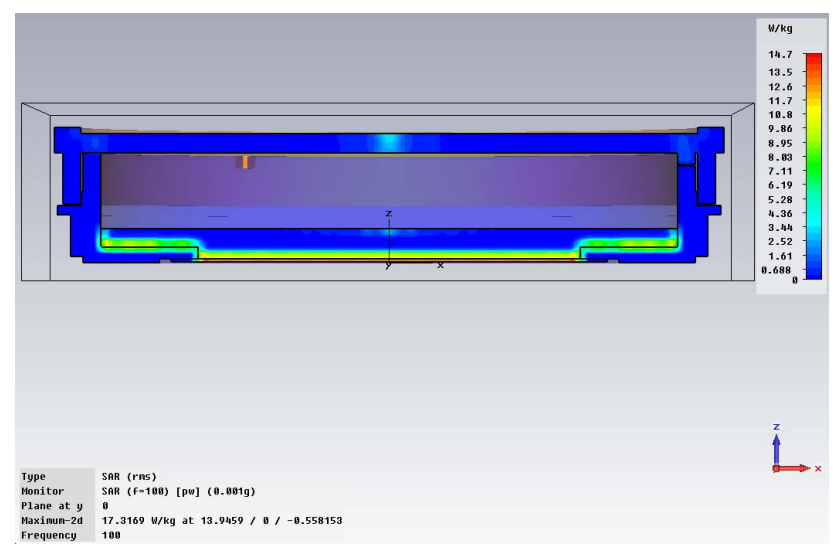

Fig. 5. Results of the SAR calculation $(0.001 \mathrm{~g} \mathrm{RMS})$ at $100 \mathrm{GHz}$ in the cell container for a plane wave with $2 \mathrm{~mW} / \mathrm{cm}^{2}$ travelling in the $\mathrm{z}$-direction. The cut plane is a cross section through the center. Results are valid both for isothermic and open boundary conditions.

stricted to one core) took about two weeks, each. Therefore, initial calculations had to concentrate on the lowest frequency of $100 \mathrm{GHz}$ used for the field exposition experiments. Due to program restrictions the excitation had to be simplified assuming a planar wave of given power flux density applied from below. The three dimensional model of the sample container is shown in Fig. 1b). Both, cell layer and DMEM culture medium are assumed to have similar properties and have been modelled with $\varepsilon_{r}=5.98$ and $\tan \delta=1.83$ using the dielectric properties measured for the culture medium using THz time-domain spectroscopy (Pupeza et al., 2007). Dish, bottom foil and lid of the sample container are modelled using the dielectric properties specified by the manufacturer for $100 \mathrm{GHz}$ (dish: $\varepsilon_{r}=2.28$ and $\tan \delta=0.0001$, foil: $\varepsilon_{r}=2.34$ and $\tan \delta=0.005$, lid: $\varepsilon_{r}=3$ and $\tan \delta=0.01$ ). For the lateral surfaces, open boundary conditions have been chosen, always, since the sample container is surrounded by a heat-insulating Rohacell ${ }^{\mathrm{TM}}$ foam. In contrast to this, top and bottom of the sample container are modelled with open or isothermic boundary conditions corresponding to a good thermal insulation or good heat transfer by convection of the surrounding air, respectively.

The calculated SAR distributions turn out to be identical for both open and isothermic boundary conditions which is consistent with the SAR value representing the power absorption, only. Figures 2 and 3 show the SAR distributions at $100 \mathrm{GHz}$ in two perpendicular cut planes after irradiation of the sample container from below with a homogeneous wave having a power flux density of $10 \mathrm{~mW} / \mathrm{cm}^{2}$. The SAR distributions represent RMS averages over very small sample volume masses of $0.001 \mathrm{~g}$. For this excitation, the maximum SAR value in the layer of the cells reaches values up to $66.3 \mathrm{~W} / \mathrm{kg}$. Since this is much above a value of $2 \mathrm{~W} / \mathrm{kg}$ considered to cause negligible heating during local body exposition at lower frequencies (ICNIRP, 1998) we 


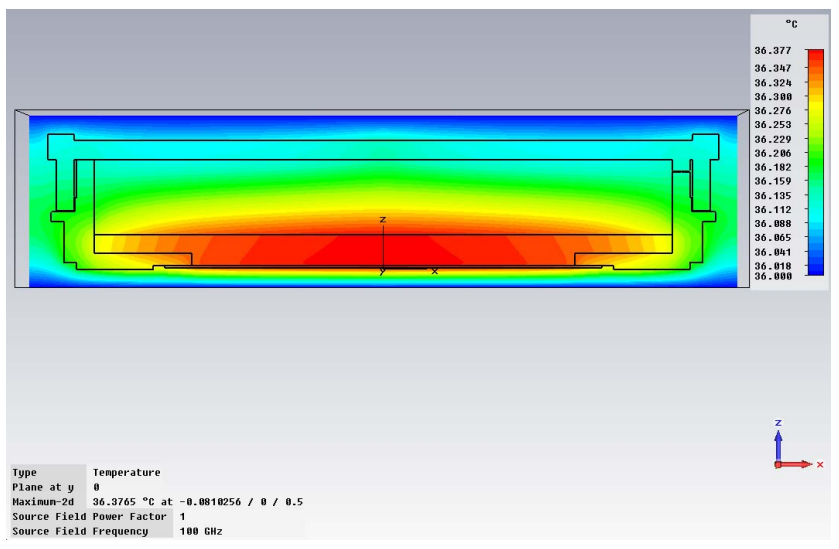

Fig. 6. Results of the heat distribution calculation at $100 \mathrm{GHz}$ in the cell container for a plane wave with $2 \mathrm{~mW} / \mathrm{cm}^{2}$ travelling in the z-direction. The cut plane is a cross section through the center. Isothermic boundary conditions have been chosen above and underneath the sample container.

expect considerable heating of the cells. Field exposition experiments above the power flux density limit were therefore performed at $2 \mathrm{~mW} / \mathrm{cm}^{2}$ to exclude possible thermal effects to influence the outcome of the experiments. Figures 4 and 5 show the SAR calculation for this power flux density. The maximum SAR value is now reduced to $13.34 \mathrm{~W} / \mathrm{kg}$. Within the numerical accuracy the maximum SAR value scales linearly with the applied power flux density.

While the SAR calculation is independent of the boundary conditions chosen for bottom and lid, the temperature distribution in the cell container will strongly depend on them. Figure 6 shows the temperature distribution in a cross section for the isothermic case, whereas Fig. 7 shows the results for the open boundary conditions, both for an excitation of $2 \mathrm{~mW} / \mathrm{cm}^{2}$. While the calculation with isothermic boundary conditions predicts a maximum temperature rise of $0.38 \mathrm{~K}$ (incubator temperature was considered to be $36^{\circ} \mathrm{C}$ ), the calculations with open boundary condition predicts up to $1.85 \mathrm{~K}$.

\section{Verification measurements}

In order to verify the predictions of the model, we performed measurements of the time-dependent temperature in the DMEM culture medium during exposition using a metal-free and fiber-coupled electro-optic thermometer Fotemp4 (OptoCon, Dresden, Germany) and the probe TK5/2 $(0.55 \mathrm{~mm}$ thickness) already used in a similar context, before (Schrader et al., 2008). Figure 8 shows time traces measured for two different power flux densities of approximately $30 \mathrm{~mW} / \mathrm{cm}^{2}$ and $3 \mathrm{~mW} / \mathrm{cm}^{2}$. The incubator temperature was set to $37^{\circ} \mathrm{C}$ in this case. Both experiments show that the culture medium heats up from room temperature to a steady-state value well

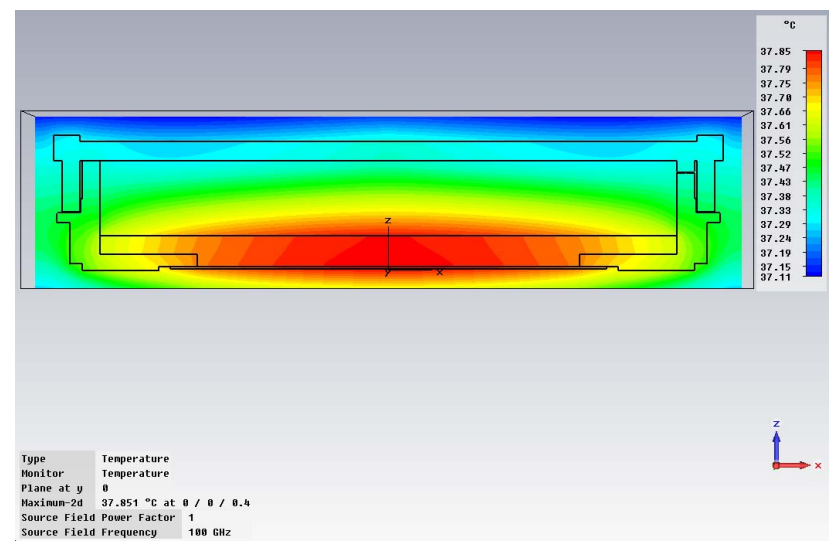

Fig. 7. Results of the heat distribution calculation at $100 \mathrm{GHz}$ in the cell container for a plane wave with $2 \mathrm{~mW} / \mathrm{cm}^{2}$ travelling in the $\mathrm{z}$ direction. The cut plane is a cross section through the center. Open boundary conditions have been chosen above and underneath the sample container.

above the incubator temperature that depends on the power flux density. For the power flux density of $3 \mathrm{~mW} / \mathrm{cm}^{2}$ the $\mathrm{THz}$ beam was blocked temporarily during the experiment causing the temperature to decrease to the incubator temperature.

In Fig. 8 the acceptable temperature range between $36^{\circ} \mathrm{C}$ and $40^{\circ} \mathrm{C}$ in which no detrimental effect on the cell growth is expected is indicated as hatched area. The temperature curve in Fig. 8 indicates a temperature below $30^{\circ} \mathrm{C}$ when the experiment is started. This is due to the fact that the sample had to be removed from another incubator before being measured in the exposition chamber. It can be seen, that a power flux density of approximately $30 \mathrm{~mW} / \mathrm{cm}^{2}$ during exposure leads to overheating of the cells, whereas a power flux density of $3 \mathrm{~mW} / \mathrm{cm}^{2}$ causes the temperature to stay in the acceptable range. In the case of irradiation with $3 \mathrm{~mW} / \mathrm{cm}^{2}$ the steadystate value of the temperature lies approximately $2.5 \mathrm{~K}$ above the incubator temperature. Compared to the calculation results shown in Figs. 6 and 7, this indicates that the model is valid.

\section{Conclusions}

In this contribution we have shown the experimental setups, the working plan and first dosimetric calculations for a comprehensive study on possible genotoxic effects of $\mathrm{THz}$ radiation as initiated by the German Federal Office for Radiation Protection. The dosimetric calculations show that SAR values up to $13.34 \mathrm{~W} / \mathrm{kg}$ and a heating of the cell by $1.85 \mathrm{~K}$ have to be expected when irradiating the cell containers with $2 \mathrm{~mW} / \mathrm{cm}^{2}$. However, these values represent upper limits since the illumination of the samples with a Gaussian beam will transfer less heat into the sample and the sample 


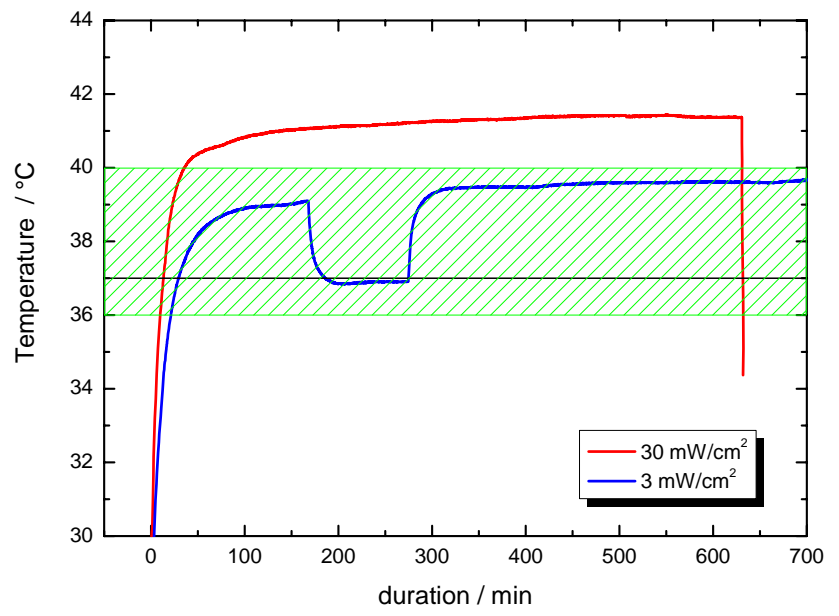

Fig. 8. Measurements of the time dependent temperature in the culture medium for two different irradiation power densities of approximately $30 \mathrm{~mW} / \mathrm{cm}^{2}$ and $3 \mathrm{~mW} / \mathrm{cm}^{2}$ using a fibre-optic semiconductor thermometer. The room-temperature sample has been put into the incubator set at $37^{\circ} \mathrm{C}$. For $3 \mathrm{~mW} / \mathrm{cm}^{2}$ the $\mathrm{THz}$ beam was blocked temporarily during the experiment. The hatched area indicates the temperature region in which no detrimental effect on the cell growth is expected.

container is not thermally insulated to the surrounding air as assumed by open boundary conditions. Temperature measurements in the culture medium during exposition show that the dosimetric model approximates realistic results. Field expositions are currently underway. Results of the experiments are expected in 2010.

Acknowledgements. The authors would like to thank the project team that is working closely together to perform the field exposition experiments: B. Heinen, K. Baaske, and M. Koch, all with the Institut für Hochfrequenztechnik at Technical University Braunschweig for performing the field exposition experiments above $600 \mathrm{GHz}$ and H. Hintzsche and H. Stopper from the Department of Toxicology at the University of Würzburg and U. Kärst and J. Wehland from the Helmholtz Centre for Infection Research in Braunschweig for preparation and evaluation of the biological samples.

Furthermore the authors would like to thank E. Schmid from the Institute for Cell Biology at University Munich and A. Enders from the Institute for Electromagnetic Compatibility at Technical University Braunschweig for scientific advice.

\section{References}

ANSI Z136.1-2007, American National Standard for Safe Use of Lasers, 2007.

Berry, E., Walker, G. C., Fitzgerald, A. J., Zinov'ev, N. N., Chamberlain, M., Smye, S. W., Miles, R. E., and Smith, M. A.: Do invivo terahertz imaging systems comply with safety guidelines?, J. Laser Appl., 15, 192-198, 2002.

CST Microwave Studio, http://www.CST.de/, 2009.
ICNIRP Guidelines for limiting exposure to time varying electric, magnetic, and electromagnetic fields (up to $300 \mathrm{GHz}$ ), Health Phys., 74, 494-522, 1998.

Kleine-Ostmann, T., Münter, K., Spitzer, M., and Schrader, T.: The electromagnetic environment above $100 \mathrm{GHz}$ : Electromagnetic compatibility, personal safety and regulation issues, Joint 31st International Conference on Infrared and Millimeter Waves and 14th IEEE International Conference on $\mathrm{THz}$ Electronics (IRMMW-THz 2006), Shanghai, China, 2006.

Kleine-Ostmann, T., Schrader, T., Bieler, M., Siegner, U., Monte, C., Gutschwager, B., Hollandt, J., Steiger, A., Werner, L., Müller, R., Ulm, G., Pupeza, I., and Koch, M.: THz Metrology, Frequenz, special issue on Terahertz Technologies and Applications, 62, 137-148, 2008.

Kleine-Ostmann, T., Jastrow, C., Salhi, M., Schrader, T., Hintzsche, H., Stopper, H., Kärst, U., Heinen, B., Baaske, K., and Koch, M.: In Vitro Field Exposition of Skin Cells between $100 \mathrm{GHz}$ and 2.52 THz, Procedings of the 34th International Conference on Infrared, Millimeter and Terahertz Waves (IRMMW-THz 2009), Busan, Korea, 2009.

Korenstein-Ilan, A., Barbul, A., Hasin, P., Eliran, A., Gover, A., and Korenstein, R.: Terahertz Radiation Increases Genomic Instability in Human Lymphocytes, Rad. Research, 170, 224-234, 2008.

Matter, B. and Schmid, W.: Trenimon-induced chromosomal damage in bone-marrow cells of six mammalian species, evaluated by the micronucleus test, Mutat. Res., 12, 417-425, 1971.

NiSG-Gesetz zum Schutz vor nichtionisierender Strahlung bei der Anwendung am Menschen (NiSG), Bundesgesetzblatt Jahrgang 2009 Teil I Nr. 49, 2433-2435, 2009.

Physical Agents Directive 2004/40/EC: Directive of the European Parliament and of the Council on the minimum health and safety requirements regarding the exposure of workers to the risks arising from physical agents (electromagnetic fields), 2004.

Piesiewicz, R., Kleine-Ostmann, T., Krumbholz, N., Mittleman, D., Koch, M., Schöbel, J., and Kürner, T.: Short-Range Ultra Broadband Terahertz Communications: Concept and Perspectives, IEEE Antenn. Propag. M., 49, 24-39, 2007.

Pupeza, I., Wilk, R., and Koch, M.: Highly Accurate Optical Material Parameter Determination with $\mathrm{THz}$ Time Domain Spectroscopy, Opt. Express, 15, 4335-4350, 2007.

Schrader, T., Münter, K., Kleine-Ostmann, T., and Schmid, E.: Spindle Disturbances in Human-Hamster Hybrid (AL) Cells Induced by Mobile Communication Frequency Range Signals, Bioelectromagnetics, 29, 626-639, 2008.

Siegel, P. H.: THz Technology, IEEE Trans. Microwave Theory Tech. 50th Anniversary Issue, 50, 910-928, 2002.

Singh, N. P., McCoy, M. T., Tice, R. R., and Schneider, E. L.: A simple technique for quantitation of low levels of DNA damage in individual cells, Exp. Cell Res., 175, 184-191, 1988.

Thomas Keating Instruments, http://www.terahertz.co.uk/, 2009.

THz-Bridge: Tera-Hertz radiation in Biological Research, Investigation on Diagnostics and study of potential Genotoxic Effects, Final Report, http://www.frascati.enea.it/THz-BRIDGE, 2004.

Werner, L., Hübers, H.-W., Meindl, P., Müller, R., Richter, H., and Steiger, A.: Towards traceable radiometry in the terahertz region, Metrologia, 46, 160-164, 2009. 